\title{
Design of a Neuronal Array
}

\author{
Bart G. Borghuis, ${ }^{1}$ Charles P. Ratliff, ${ }^{2}$ Robert G. Smith, ${ }^{1}$ Peter Sterling, ${ }^{1}$ and Vijay Balasubramanian ${ }^{2,3}$ \\ Departments of ${ }^{1}$ Neuroscience and ${ }^{2}$ Physics and Astronomy, University of Pennsylvania, Philadelphia, Pennsylvania 19104-6396, and ${ }^{3}$ School of Natural \\ Sciences, Institute for Advanced Study, Princeton, New Jersey 08540
}

Retinal ganglion cells of a given type overlap their dendritic fields such that every point in space is covered by three to four cells. We investigated what function is served by such extensive overlap. Recording from pairs of ON or OFF brisk-transient ganglion cells at photopic intensities, we confirmed that this overlap causes the Gaussian receptive field centers to be spaced at $\sim 2$ SDs $(\sigma)$. This, together with response nonlinearities and variability, was just sufficient to provide an ideal observer with uniform contrast sensitivity across the retina for both threshold and suprathreshold stimuli. We hypothesized that overlap might maximize the information represented from natural images, thereby optimizing retinal performance for many tasks. Indeed, tested with natural images (which contain statistical correlations), a model ganglion cell array maximized information represented in its population responses with $\sim 2 \sigma$ spacing, i.e., the overlap observed in the retina. Yet, tested with white noise (which lacks statistical correlations), an array maximized its information by minimizing overlap. In both cases, optimal overlap balanced greater signal-to-noise ratio (from larger receptive fields) against greater redundancy (because of larger receptive field overlap). Thus, dendritic overlap improves vision by taking optimal advantage of the statistical correlations of natural scenes.

Key words: retina; ganglion cell; paired recording; contrast sensitivity; ideal observer; natural scenes; optimal coding

\section{Introduction}

There is a well studied hypothesis for the function of the receptive field of a ganglion cell: the center Gaussian sums locally correlated signals to improve the signal-to-noise ratio (SNR) (Tsukamoto et al., 1990), and the surround Gaussian subtracts broadly correlated signals to reduce redundancy (Barlow, 1961; Srinivasan et al., 1982; Laughlin, 1983; Atick and Redlich, 1990; van Hateren, 1992, 1993). Each cell type forms an array, whose spacing sets spatial resolution. Array structure is such that the nearest neighbors of a cell lie at the perimeter of its dendritic arbor (Wassle and Boycott, 1991), and this causes dendritic fields to overlap considerably. In fact, despite the common notion that cells in an array simply "tile" (Emoto et al., 2006), the "coverage factor" from dendritic overlap is actually 3-4 (Wassle, 2004). Because synapses distribute on the membrane at a constant density (Freed et al., 1992; Kier et al., 1995; Calkins and Sterling, 2007), dendritic overlap uses many more synapses than would simple tiling. Although this costly design is conserved across cell types and species, its function is unknown.

One idea is that dendritic overlap is just sufficient to produce spatially uniform contrast sensitivity across the array. Receptive field centers in the dark-adapted rabbit retina are separated by

Received July 23, 2007; revised Feb. 7, 2008; accepted Feb. 7, 2008.

This work was supported by National Institutes of Health (NIH) Grants EY000828, EY008124, and EY016607 and National Science Foundation Grant IBN-0344678. C.P.R. was supported in part by NIH Vision Training Grant T3207035. V.B. was supported in part as the Helen and Martin Chooljian Member of the Institute for Advanced Study. We thank Narender Dhingra for his generous tutelage early in the project. We also thank Jonathan Demb, Liam Paninski, and Simon Laughlin for comments.

Correspondence should be addressed to Bart G. Borghuis, Department of Neuroscience, 123 Anatomy/Chemistry Building, University of Pennsylvania, Philadelphia, PA 19104. E-mail: bart@retina.anatomy.upenn.edu. D01:10.1523/JNEUROSCI.5259-07.2008

Copyright $\odot 2008$ Society for Neuroscience $\quad 0270-6474 / 08 / 283178-12 \$ 15.00 / 0$ $\sim 2$ SDs $(2 \sigma)$ DeVries and Baylor (1997) and by $2-3 \sigma$ in lightadapted primate retina (Chichilnisky and Kalmar, 2002, 2003; Frechette et al., 2005; Shlens et al., 2006; Field and Chichilnisky, 2007). Two $\sigma$ is the largest separation at which Gaussians sum linearly to form a flat surface (DeVries and Baylor, 1997), but given inhibitory surrounds, nonlinearities, and response variability, the question remained: does this center spacing actually achieve uniform sensitivity? To answer this, we recorded from pairs of neighboring ganglion cells and asked an ideal observer (Geisler et al., 1991; Dhingra et al., 2003) to detect a spot based on the responses of either cell alone or both combined. Array performance indeed proved to be spatially uniform.

Although spot detection is a special case, performance for any task is limited by the amount of represented information (Geisler, 1989; Cover and Thomas, 1991; Thomson and Kristan, 2005). Therefore, we assessed performance more broadly by measuring how the amount of information represented by an array depends on its structure. Larger receptive fields increase information by collecting more cone inputs and thus improving SNR. However, at fixed spacing, larger receptive fields also overlap more, which increases redundancy in an array. Because the response range of a cell is finite, redundancy reduces the total information of an array. Thus, we hypothesized that receptive field overlap should balance the advantage of greater SNR against the disadvantage of greater redundancy. We found for model arrays that natural images (whose statistical correlations, when averaged, can improve SNR) are optimally represented by $\sim 2 \sigma$ spacing; however, white noise (which lacks statistical correlations) is optimally represented by simple tiling without overlap. Thus, overlap in a ganglion cell array cooperates with single-cell synaptic weighting to optimally represent the statistical structure of natural scenes. 


\section{Materials and Methods}

Preparation. We recorded responses from brisk-transient ganglion cell pairs in the guinea pig retina in vitro. Retinas were obtained from adult guinea pigs (400-700 g) of either sex. An animal was first deeply anesthetized (ketamine at $40 \mathrm{mg} / \mathrm{kg}$, xylazine at $5 \mathrm{mg} / \mathrm{kg}$, and pentobarbital at $50 \mathrm{mg} / \mathrm{kg}$ ); then its eyes were removed, and the animal was killed by intracardiac injection of barbiturate (pentobarbital at $20 \mathrm{mg} / \mathrm{kg}$ ). Eyes were placed in oxygenated $\left(95 \% \mathrm{O}_{2}-5 \% \mathrm{CO}_{2}\right)$ Ames medium (Sigma, St. Louis, MO) containing sodium bicarbonate $(1.9 \mathrm{~g} / \mathrm{L})$ and glucose $(3.6$ $\mathrm{g} / \mathrm{L}$ ). The anterior half and vitreous humor of each eye were removed, and the posterior half was radially incised and mounted on filter paper with ganglion cell layer up, preserving the attachment of the retina to pigment epithelium, choroid, and sclera. The whole-mount retina preparation was then placed in a recording chamber on an upright micro-

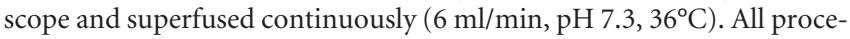
dures conformed to National Institutes of Health and University of Pennsylvania guidelines.

Recording. Viewing under infrared-Hoffman optics, we selected adjacent brisk-transient cells based on soma size $(\sim 20 \mu \mathrm{m})$ and spacing $(\sim 200 \mu \mathrm{m})$. Simultaneous recordings were obtained via loose seals with independently manipulated glass electrodes $(8-12 \mathrm{M} \Omega)$. Stimuli were computer generated (Pelli, 1997), displayed with a miniature cathode ray tube monitor (MicroBrightField, Colchester, VT), and projected on the retina through the microscope optics $(4 \times$; numerical aperture 0.13 ; image size, $3.2 \times 2.4 \mathrm{~mm}$ ). Mean background intensity was 7900 photons/ $\mu \mathrm{m}^{2} / \mathrm{s}$ (equivalent to $\sim 2.0 \times 10^{4} \mathrm{R}^{\star} /$ cone $/ \mathrm{s}$ and $\sim 0.6 \times 10^{4} \mathrm{R}^{*} / \mathrm{rod} / \mathrm{s}$ at $535 \mathrm{~nm}$ or $36.1 \mathrm{~cd} / \mathrm{m}^{2}$, using a measured nodal distance of $5.05 \mathrm{~mm}$ and pupil diameter of $2.0 \mathrm{~mm}$ ). The same retinal illuminance for cat or primate is equivalent to $\sim 100 \mathrm{~cd} / \mathrm{m}^{2}$. In primates, this intensity saturates the rod photoreceptors and is therefore considered photopic (Penn and Hagins, 1972; Adelson, 1982). A recent study, however, showed that guinea pig rods at this light level are not saturated but instead account for $50-70 \%$ of the total response (Yin et al., 2006). Similar mesopic rod adaptation has been demonstrated in other species with cone-sparse retinas (Tamura et al., 1989; Nakatani et al., 1991). Defining "photopic" as the intensity range at which the rods are saturated, our mean background intensity is formally mesopic.

Spatiotemporal receptive fields were measured with a binary white noise (checkerboard) stimulus (DeAngelis et al., 1995; Chichilnisky, 2001). Cells were confirmed as brisk transient based on their spatiotemporal response characteristics and autocorrelogram (DeVries and Baylor, 1997; Chichilnisky and Kalmar, 2002; Koch et al., 2004). Reverse correlograms were computed on-line and fitted with difference-of-Gaussians functions to locate the receptive field centers and relative surround strength and width. Quality of the fits was evaluated by normalizing the receptive field peak to 1 and computing the root mean square error (RMSE)

$$
\mathrm{RMSE}=\sqrt{\sum\left(\bar{X}_{i, j}-X_{i, j}\right)^{2}}
$$

between the data points and the fitted function. For all cells, RMSE was low: $0.08 \pm 0.01$ (mean \pm SEM; $n=44$ ). There was no difference between the quality of fits for ON and OFF cells (ON, $0.08 \pm 0.012, n=22$; OFF, $0.08 \pm 0.010, n=30$ ). Optimal spot size was determined for each cell from the response to a brief spot centered on the receptive field (diameter of 50-2000 $\mu \mathrm{m}, 100 \mathrm{~ms}$ ). Optimal spot size for members of a neighboring pair typically differed by $<5 \%$ and never $>10 \%$. In the detection experiments, the applied spot size was the average of the optima of two cells.

To measure contrast detection, a spot was presented for $100 \mathrm{~ms}$. Weber contrast

$$
C=\left(I_{s}-I_{b}\right) / I_{b} \text {, }
$$

where $I_{s}$ is spot intensity and $I_{b}$ is background intensity, was varied between $0.5 \%$ and $30 \%$ and comprised an intensity increment (light spot) for ON cells and a decrement (dark spot) for OFF cells. Recordings were analyzed only when responsiveness for both cells was stable for 200 trials. To minimize adaptation, stimuli were presented in a random, interleaved order, and each $100 \mathrm{~ms}$ flash was followed by $900 \mathrm{~ms}$ of background level illumination.

After recording, cells were penetrated with a sharp electrode and filled with Neurobiotin and fluorescent dyes of contrasting color (Alexa Fluor 488 and 568; Invitrogen, Carlsbad, CA). Tissue was then fixed (20 min; $4 \%$ paraformaldehyde), reacted with cyanine 5 -conjugated streptavidin, mounted on a glass slide, and imaged by confocal microscopy $(60 \times$, numerical aperture 0.9 ).

Contrast detection. To measure the contrast sensitivity surface, detection thresholds of the recorded ganglion cell pairs were computed with an ideal observer (Geisler et al., 1991; Dhingra et al., 2003; Dhingra and Smith, 2004). Briefly, responses were binned (40 ms) (Dhingra and Smith, 2004), and half of the 200 recorded trials were used to train the ideal observer, i.e., to build probability distributions for the response of each cell to each contrast. The other half was used as test trials to measure discrimination performance. We used $40 \mathrm{~ms}$ time bins because these were found to be optimal in a previous study of contrast sensitivity that used the same guinea pig brisk-transient cells (Dhingra et al., 2003). Shorter bins required more stimulus repeats without improving detection threshold, whereas longer bins degraded detection (Dhingra et al., 2003, their Fig. 4 B). In responses to bright stimuli, variance of first spike time and duration of the response to a $100 \mathrm{~ms}$ flash is $\ll 40 \mathrm{~ms}$, but, for dim flashes, variance and response duration increases significantly. It appears that $40 \mathrm{~ms}$ time bins optimally account for these two factors.

Combining the responses of two cells, the ideal observer was provided with twice the number of temporal bins. We used a "template method," which maximizes performance (percentage correct) by comparing the spike count in time bins of a test trial to response probability distributions obtained from the training trials. Decisions were based on a likelihood ratio test that took the stimuli (spot/no spot) to be equally likely a priori, reflecting the experimental design. This test is optimal for noise distributions (i.e., conditional probabilities of the response given stimulus) of any shape (Dhingra and Smith, 2004). Our procedure is equivalent to a Bayesian discriminant, or maximum likelihood method for stimuli occurring with equal probability (Braverman, 1962; Geisler et al., 1991; Duda et al., 2000).

We tested several discrimination templates, including the difference between the means (optimal when the noise does not vary), the difference between the means divided by the variance (optimal when the noise changes between time bins), and the Fisher linear discriminant (optimal when noise changes arbitrarily and may be correlated) and found that the differences in performance were minor. This agrees with the evaluation of various discriminant methods by Chichilnisky and Rieke (2005).

Optimal overlap for representing natural images. An observer's ability to decide correctly on any visual task is constrained by the amount of information available from the retina (Geisler, 1989; Thomson and Kristan, 2005). Thus, we hypothesized that, for a given cell density, receptive fields should overlap such that total transmitted information is maximized. To test this hypothesis, we constructed model arrays of twodimensional difference-of-Gaussians receptive fields and used images from a library of natural scenes (van Hateren and van der Schaaf, 1998) to ask how the array should be arranged to maximize represented information. We used images of natural scenes because they have characteristic spatial correlations (Field, 1987) that might in part determine the optimal overlap. Taking into account the specific statistical structure of the input of the visual system under natural viewing conditions has been important in explaining the structure of the spatiotemporal receptive field of an individual cell (Srinivasan et al., 1982; Laughlin, 1983; Atick and Redlich, 1990; Tsukamoto et al., 1990; van Hateren, 1993). Here, we applied a similar approach to determine how individual cells should be arranged within an array.

Modeling a ganglion cell array. The analysis measured information transmission in an array with a simple linear-nonlinear model, in which populations of ganglion cells were represented by linear center-surround filters whose outputs were rectified to mimic the spike threshold, and then mapped nonlinearly to fill the finite dynamic range of a ganglion cell. An overview of the model is shown in Figure $1 A$. Two variables determined how much information was transmitted: SNR of a ganglion cell and redundancy within the array. Increasing the receptive field size 
improved the SNR of the response of each cell but, at a fixed cell density, also increased the redundancy between cells. Our hypothesis was that, in the real retina, these tendencies are balanced.

To test this, we constructed a model in which a single image pixel represented a cone, and ganglion cell receptive fields were constructed by summing the cone signals with a difference-ofGaussians (center-surround) weighting function. Width and strength of the inhibitory surround were fixed to match the average values measured for brisk-transient ON and OFF cells. These ganglion cell receptive fields were arranged to mimic the relative densities of $\mathrm{ON}$ and OFF ganglion cells that we measured in the guinea pig retina (ON spacing, $\sim 32$ pixels; $\mathrm{OFF}$ spacing, $\sim 24$ pixels; results were robust to variations in the precise separation). This defined the spatial layout of the model array.

SNR of a cone. Our analysis required the SNR for the responses of receptive fields of widely varying size. Because receptive field size could not be freely manipulated in the real retina, we modeled ganglion cell noise as arising from variability at the cones and progressive degradation at the bipolar synapses. It is not feasible to directly measure variability at the cones in an intact retina preparation, but, for our model, it could be estimated from the literature.

The cone synaptic response function measured by Choi et al. (2005) shows a maximal sustained release of $\sim 250$ vesicles/s. The maximum release rate sets the dynamic response range of the cell, and, assuming that vesicle release is a Poisson process, the measured maximum gives $\sim 10$ distinguishable signaling levels over a $200 \mathrm{~ms}$ integration time (Choi et al., 2005). The SNR of the cone, defined here as cone signal variance divided by the cone noise variance (Cover and Thomas, 1991), is approximately the square of the number of signaling levels, i.e., $\mathrm{SNR}_{\text {cone }}$ of $\sim 100$. Note that, to comply with the conventions of Cover and Thomas (1991), used below in information calculations, SNR is based on signal and noise variance; another common convention uses SDs, in which case SNR equals the number of signaling levels, i.e., 10.

There are several caveats in applying the SNR estimated from the study by Choi et al. to our model ganglion cell array. First, the experiments of Choi et al. were performed in lizard, and mammalian vesicle release rates may be significantly higher (DeVries et al., 2006), which would increase SNR. Second, Choi et al. measured sustained release. At a given mean intensity, the sustained rate tells how much release can maximally decrease in response to a light flash. However, a dark flash could evoke a transient increase in release, which would increase SNR. Indeed, Choi et al. showed, by applying a light pulse paradigm or evoking maximal release with high $\mathrm{K}^{+}$, that cone release could be increased by up to twofold. In view of these uncertainties, we tested how much optimal cell spacing varies with cone SNR. We found that the results presented here are robust to $4 \log$ units of variation in the estimated cone SNR (see Results). This was because optimizing total information depended more on the tradeoff between SNR improvement by summation against redundancy and less on the actual value of the cone SNR.

SNR improvement. The ganglion cell receptive field sums responses from many cones, whose input noises reflect photon noise, which is uncorrelated. Consequently, SNR of a ganglion cell response improves relative to that of a single cone. If $a_{i}$ is the strength of a receptive field at photoreceptor $i, s_{i}$ the vesicle release rate at photoreceptor $i$, and $0<r_{i j} \leq$ 1 the correlation coefficients between photoreceptors, i.e., $\left\langle s_{i} s_{j}\right\rangle=s^{2} r_{i j}$, with $\left\langle s_{i}{ }^{2}\right\rangle=s^{2}$, then assuming uncorrelated noise and letting $\mathrm{SNR}_{\text {cone }}$ be the SNR of a single cone, SNR after summing over the cones in a receptive field is $f^{2} \cdot \mathrm{SNR}_{\text {cone, }}$, where
B

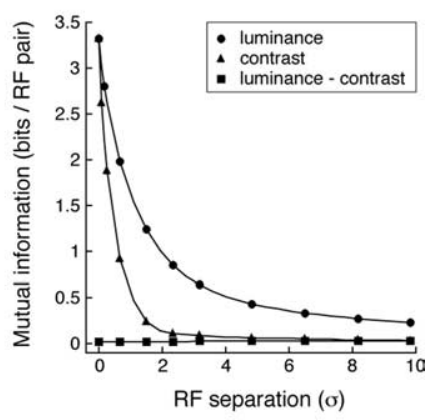
photoreceptors in the array (Tsukamoto et al., 1990). Here, $r_{i j}$ is the correlation coefficient between pixel intensities at different points. In practice, the computationally lengthy sum over pixels in the formula for $f^{2}$ was performed over an $80 \times 80$ array for model difference-of-Gaussian receptive fields with relative surround widths and gains fixed by our measurements for ON and OFF brisk-transient cells. We verified that increasing the size of the pixel array did not change the computed value of $f^{2}$ in the range of center sizes used here. Over this range, the signal-tonoise improvement factor was well described by a quadratic polynomial in $\sigma$ [ON mean square error (MSE), 0.31; OFF MSE, 0.38] (for a discussion of the origin of this quadratic dependence, see Derrington and Lennie, 1984). This polynomial was used to extrapolate the SNR improvement factor to larger $\sigma$ when necessary.

SNR of a ganglion cell. Summation over cones improves SNR in a receptive field, but noise in both the bipolar cell (which forwards the cone signal to the ganglion cell) and in the ganglion cell itself reduces it. Although cone SNR relied on an estimate, subsequent SNR reduction attributable to noise in the bipolar cell and ganglion spike generator is known. Comparing contrast sensitivity measured postsynaptic to the cones with contrast sensitivity in a ganglion cell showed a twofold reduction in contrast threshold across the bipolar cell (B. G. Borghuis, R. G. Smith, and P. Sterling, unpublished observation). This implies a twofold reduction in signaling levels and hence a fourfold reduction in SNR (defined as the ratio of signal and noise variances; see above). Furthermore, direct measurement showed that the conversion from membrane voltage to spike train raises contrast threshold by another twofold (Dhingra and Smith, 2004). This implies another fourfold reduction of SNR. For the overall SNR of a ganglion receptive field, this gives

$$
\mathrm{SNR}_{\mathrm{rf}}=(1 / 16) \times f^{2} \times \mathrm{SNR}_{\text {cone }},
$$

where $1 / 16$ represents the combined SNR reduction in the bipolar and ganglion cell, and $f^{2}$ is the receptive field SNR improvement factor 
(Tsukamoto et al., 1990) (Eq. 3). Equation 4 gives the SNR of the linear filter whose output was rectified and mapped to a response level by a saturating nonlinearity. The nonlinearity filled the bandwidth of the model cell as efficiently as possible by using as a response function the cumulative probability distribution of the rectified filter outputs given natural image inputs [also called "histogram equalization" (Laughlin, 1983)]. The resulting nonlinearity had a characteristic sigmoidal, saturating form, roughly approximating the contrast response curve of a ganglion cell (Dhingra et al., 2003). Because ON and OFF type brisktransient cells have the same contrast sensitivity (Dhingra et al., 2003), the same method was applied for both types.

We chose to model the nonlinearity rather than measure it because our analysis of optimality required contrast response functions for cells of varying size (whereas the actual arrays use cells of fixed size) and also for scenes with widely varying mean contrasts. The SNR improvement by pooling in the linear filter translates directly into SNR improvement of the model ganglion cell output because, in the linear range of the response function, noise in the filter sets the number of discriminable response levels. Because the optimal spacing turned out to depend on SNR improvement rather than the absolute SNR value, the precise functional form of the nonlinearity was unimportant to our findings. Finally, we approximated the ganglion cell output as a Gaussian channel (Atick and Redlich, 1990; Laughlin, 1994; Mante et al., 2005) so that the amount of information about visual stimuli represented by this response is (Cover and Thomas, 1991)

$$
I_{1}=(1 / 2) \times \log _{2}\left[1+\mathrm{SNR}_{\mathrm{rf}}\right] .
$$

Our results do not depend strongly on the Gaussian assumption because they are driven by two general facts: (1) the number of discriminable signaling levels increases with receptive field diameter (Derrington and Lennie, 1984), and (2) information goes as the log of the number of signaling levels (Cover and Thomas, 1991; Laughlin, 1994). Equation 5 provides an approximation that embodies these two facts.

Information in a receptive field array. Finally, the total amount of information about visual stimuli represented by an array of $N$ overlapping receptive fields is

$$
I_{N}=\rho(\sigma) \times N \times I_{1},
$$

where the factor $\rho(\sigma)$, which lies between 0 and 1 , accounts for the redundancy, or shared information, in the responses of an array of receptive fields with center SD $\sigma$. To compute $\rho(\sigma)$, we split the response of a receptive field into a weighted sum of local luminance and contrast. Luminance was defined here as the response of the central Gaussian and contrast as the difference between the responses of the center and a balanced surround (i.e., a surround with relative strength equal to 1 ; see Appendix). In natural images, luminance is correlated over large distances (Burton and Moorhead, 1987; Field, 1987), but contrast correlations decay rapidly (Fig. $1 B$ ). In addition, luminance and contrast in natural scenes are statistically independent (Mante et al., 2005) (Fig. 1 B). Using these facts, it is shown in Appendix that, for natural scenes, a large array of receptive fields signals mostly contrast information. Furthermore, because of the short range of contrast correlations, most of the redundancy in contrast responses comes from receptive field overlap. This is used in Appendix to show that the information represented per receptive field $\left(I_{N} / N=\rho(\sigma) \cdot I_{1}\right)$ approaches a constant as the array size increases, given by

$$
\rho(\sigma) \cdot I_{1}=\left(I_{1}-2 \cdot M I_{\text {adj }}-2 \cdot M I_{\text {diag }}\right) .
$$

Here $M I_{\text {adj }}$ and $M I_{\text {diag }}$ measure the mutual information (for definition, see Cover and Thomas, 1991) in the contrast responses of nearest neighbor adjacent and diagonal pairs in an array of receptive fields with center SD $\sigma$ (Cover and Thomas, 1991). Specifically, given the responses $R_{1}=$ $\left\{r_{1}\right\}$ and $R_{2}=\left\{r_{2}\right\}$ of two receptive fields, with probability distributions $\operatorname{Pr}\left(r_{1}\right)$ and $\operatorname{Pr}\left(r_{2}\right)$, and a joint distribution $\operatorname{Pr}\left(r_{1}, r_{2}\right)$, the mutual information is $M I\left(R_{1}, R_{2}\right)=H\left(R_{1}\right)+H\left(R_{2}\right)-\mathrm{H}\left(R_{1}, R_{2}\right)$, where $H$ is the entropy of a distribution given as $H(R)=-\Sigma_{r} \operatorname{Pr}(r) \log \operatorname{Pr}(r)$. This statistical quantity summarizes how well the response of one receptive field predicts the another response: every bit of mutual information improves accuracy of prediction by a factor of $\sim 2$. The response probability distributions were measured by exhaustively sampling the responses of model receptive fields to a library of natural scenes (van Hateren and van der Schaaf, 1998).

\section{Results}

We studied 26 pairs ( 8 ON/ON, 12 OFF/OFF, and 6 ON/OFF) of neighboring brisk-transient ganglion cells in the visual streak of the guinea pig retina (Demb et al., 1999). The brisk-transient cell corresponds to the parasol/M cell in primate retina (Wassle, 2004).

\section{Overlap of dendritic fields and receptive fields}

When neighboring cells of the same type (ON or OFF) were dye injected, their dendritic fields were seen to overlap (Fig. 2 A). OFF cells overlapped slightly more than $\mathrm{ON}$ cells, but generally the dendritic tips of a cell reached the soma of its neighbor. This degree of overlap gives a "dendritic coverage factor" (dendritic field area $\times$ cell density) of $\sim 3.5$ and agrees with previous studies that filled cells with fluorescent dye or Neurobiotin (Vaney, 1991; Dacey and Brace, 1992; Lohmann and Wong, 2001).

To measure receptive field overlap, we recorded simultaneously from neighboring cells while stimulating with spatiotemporal white noise (Fig. 2B). Receptive fields were fitted with a radial difference-of-Gaussians function:

$$
\text { Receptive field }=L_{c}-k L_{s},
$$

where $L_{c}$ is a unit normalized Gaussian representing the excitatory center, $L_{s}$ is a unit normalized Gaussian representing the inhibitory surround, and $k$ represents the relative strength of the surround. The average center width (SD of the central Gaussian, $\sigma)$ was $111 \pm 26 \mu \mathrm{m}(\mathrm{ON}$, mean $\pm \mathrm{SD} ; n=22)$ and $116 \pm 30 \mu \mathrm{m}$ (OFF; $n=30$ ). The relative surround width (surround SD in units of center $\sigma)$ was $1.37 \pm 0.44 \sigma(\mathrm{ON} ; n=22)$ and $1.40 \pm 0.45$ $\sigma(\mathrm{OFF} ; n=30)$. The relative surround strength (i.e., $k$ is weighting of the surround Gaussian) was $0.81 \pm 0.31(\mathrm{ON} ; n=22)$ and $0.72 \pm 0.34$ (OFF; $n=30)$. Absolute distance between nearest neighbor receptive field centers was $228 \pm 85 \mu \mathrm{m}$ (ON/ON; $n=$ $8)$ and $191 \pm 56 \mu \mathrm{m}(\mathrm{OFF} / \mathrm{OFF} ; n=12)$. Thus, receptive field centers were spaced at $2.05 \pm 0.50 \sigma(\mathrm{ON} / \mathrm{ON})$ and $1.86 \pm 0.55 \sigma$ (OFF/OFF). OFF cells were more closely spaced, but both types were separated by $\sim 2 \sigma$.

The diameter of the excitatory region of the receptive field, measured with spots of increasing size, showed spatial summation up to $519 \pm 81 \mu \mathrm{m}(\mathrm{ON} ; n=22)$ and $487 \pm 73 \mu \mathrm{m}(\mathrm{OFF}$; $n=30)$, equivalent to $4.7 \pm 0.7 \sigma(\mathrm{ON})$ and $4.1 \pm 0.6 \sigma(\mathrm{OFF})$. As expected, functional coverage exceeded morphological coverage (Peichl and Wassle, 1979). This gave a "receptive field coverage factor" (receptive field area $\times$ cell density) of $4.1(\mathrm{ON})$ and 6.1 (OFF), which exceeds by many fold the requirements for "perfect tiling," in which the excitatory regions of receptive fields cover without overlap.

\section{Computing the sensory surface from linear summation}

Receptive fields in dim light are well described as single Gaussians, and, given their spacing at $2 \sigma$, they sum linearly to give a sensory surface that is flat (DeVries and Baylor, 1997). However, receptive fields in bright light are difference-of-Gaussians, so we calculated how the spacing of difference-of-Gaussians would affect the linearly summed sensory surface. Starting with the recorded receptive fields, we simulated arrays with different spacings and then Fourier transformed the sensory surface to assess flatness. Finally, we compared the simulated array sensitivity 

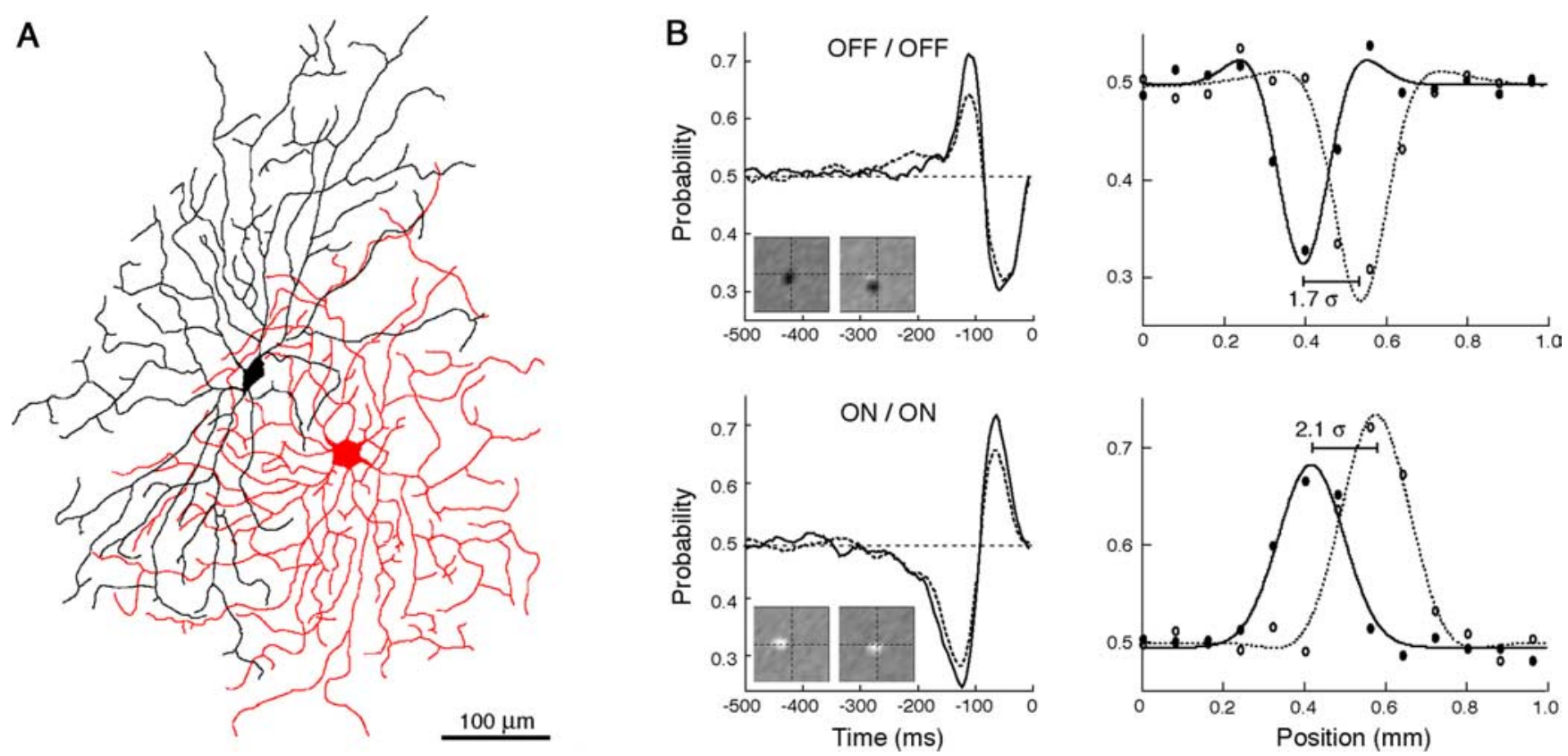

Figure 2. Dendritic fields and receptive field centers of brisk-transient ganglion cells overlap. $A$, Dendritic fields of an OFF/OFF pair typically overlapped by $\sim 40 \%$. To compute overlap, we measured the dendritic field area of each cell from a polygon drawn around the dendritic tips of a cell. We then divided the shared dendritic field area by the average dendritic field area of the two cells. $\boldsymbol{B}$, For each recorded pair, temporal filter characteristics (left; spike-triggered average of the white-noise response) were strongly similar. Spatial response profiles (right) fitted with difference-of-Gaussians functions (RMSE, $0.080 \pm 0.01$; mean $\pm \mathrm{SEM} ; n=52$ ) show that neighboring ganglion cell receptive fields overlap substantially. The example cell spacings shown here, $2.1 \sigma(0 \mathrm{~N} / \mathrm{ON})$ and $1.7 \sigma(\mathrm{OFF} / \mathrm{OFF})$, are representative for our sample and correspond, respectively, to receptive field coverage factors of 4.1 and 6.1 .

(height of the sensory surface) with that of a single cell (peak of single-cell receptive field) (Fig. 3).

In a simulation, narrow spacing (center separation $<1 \sigma$ ) produced a flat sensory surface, with an array sensitivity more than twice that of a single cell (Fig. $3 A$, top). Wider spacing decreased the array sensitivity until $\sim 2 \sigma$, where it approximately equaled single-cell sensitivity, and the surface remained flat (Fig. 3B). Still wider spacing reduced the array sensitivity below that of a single cell and caused sensitivity to dip between neighbors (Fig. 3A, bottom). The abrupt appearance of dips in the sensory surface at separations exceeding $2 \sigma$ was seen most clearly in plots of the first harmonic response (Fig. 3C). This confirmed for difference-ofGaussians receptive fields the result reported for simple Gaussians (DeVries and Baylor, 1997).

If ganglion cell receptive fields were indeed linear, then simply summing the receptive fields in an array as in Figure 3 would measure the average population response. However, the spike response of a ganglion cell is not a linear function of the stimulus. Moreover, the response varies across trials and may be redundant between neighbors. The magnitude of these factors is not known, and therefore array sensitivity cannot be computed a priori. Thus, we directly measured contrast sensitivity across the retinal surface.

\section{Measuring the sensitivity surface}

A simultaneously recorded brisk-transient ganglion cell pair was stimulated with a brief spot (100 ms, 1-15\% Weber contrast; see Materials and Methods) at nine positions along a line through the receptive field centers of cells. The linear summation of receptive fields together with assumed response linearity predicts that the combined firing rate of the pair of cells should be independent of spot position. However, we found that, for a suprathreshold stimulus, the combined firing rate actually peaked for a spot midway between the two receptive field centers (Fig. $4 \mathrm{~A}$ ). This was true for all recorded pairs $(n=11)$ (Fig. $4 B)$. We next asked how these extra spikes affected detection by an ideal observer that used all the information available from both spike trains.

The ideal observer (as detailed in Materials and Methods) detected a spot in a single-interval, two-alternative, forced-choice paradigm based on the time-binned response rates of either cell alone or the two combined (Geisler et al., 1991; Dhingra et al., 2003; Dhingra and Smith, 2004). For a suprathreshold spot over cell 1, detection performance based on the response of that cell was high ( $\sim 90 \%$ correct $)$. As the spot moved away from the center, performance declined in accordance with the domed receptive field profile (Fig. 4C). However, when responses of both cells were combined, performance did not decline for locations between the two cells (population average shown in Fig. 4D), nor did it improve as might have been expected from the increased combined response rate. Thus, although the total population firing rate varied with stimulus position (Fig. $4 A, B$ ), for a suprathreshold stimulus, the contrast sensitivity surface was flat (Fig. $4 C, D)$. Naturally, for spots beyond the receptive field centers of cells, combined sensitivity decreased because the response of the nearest neighbor was not recorded (Fig. $4 C$, positions $<100 \mu \mathrm{m}$ and $>120 \mu \mathrm{m})$.

Next, we measured the contrast sensitivity surface near detection threshold. For this, we recorded from ganglion cell pairs while a low-contrast spot of optimal size was presented in one of three locations: centered on either cell or between the two. To compute contrast detection threshold at each location, an ideal observer decided on the basis of the response of either cell alone or in combination if a spot had been presented. This paradigm is analogous to that for psychophysical detection, and the criterion for detection threshold was set accordingly at $68 \%$ correct (Dhingra et al., 2003; Dhingra and Smith, 2004). For the example pair shown in Figure $5 A-C$, a spot centered over cell 1 gave a contrast threshold of $4 \%$, and, over cell 2 , it gave $7 \%$. For a spot centered 
A
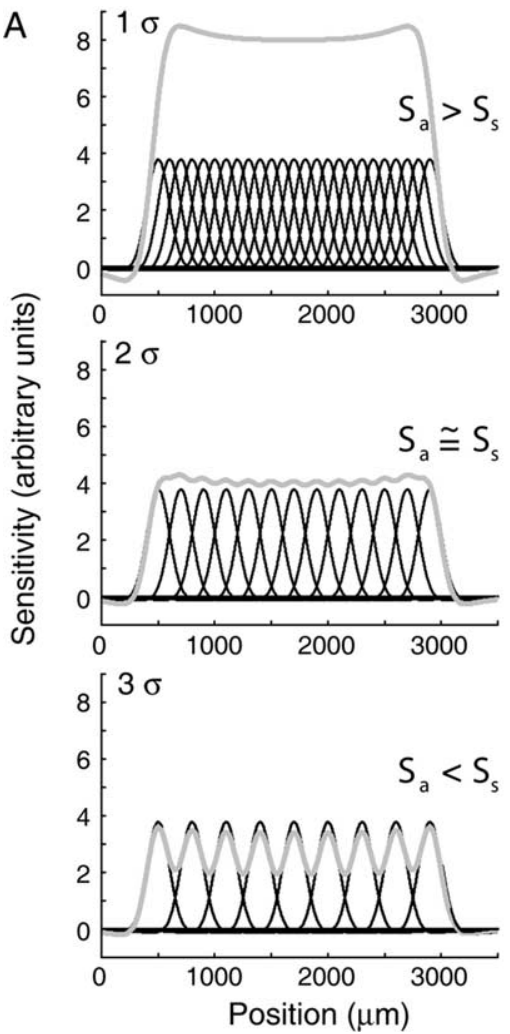

B

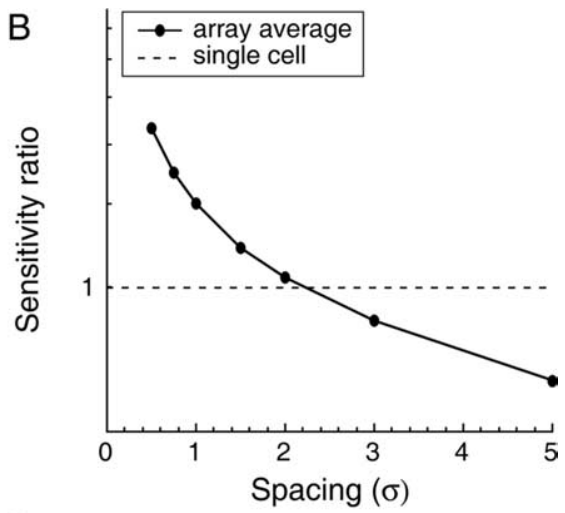

C

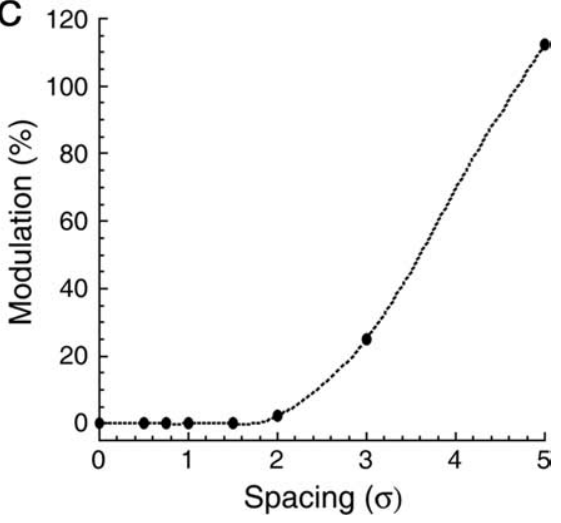

Figure 3. Simulation predicts that $2 \sigma$ receptive field center spacing gives a spatially invariant sensory surface. $\boldsymbol{A}$, The average array sensitivity (linear sum of single cell receptive fields at each location, $S_{a}$ ) at $2 \sigma$ spacing equals the peak sensitivity of a single cell (peak height of receptive field, $S_{s}$ ). Simulated difference-of-Gaussians receptive fields (black lines) show that array sensitivity (gray line) decreases with wider spacing. Receptive field parameters were obtained from spatiotemporal white-noise recordings; results shown for $0 \mathrm{~N}$ cells, $0 \mathrm{FF}$ cell parameters gave similar results. $\boldsymbol{B}$, At $\sim 2 \sigma$ spacing, average array sensitivity approximates peak sensitivity of a single cell. For spacing $>2 \sigma$, sensitivity dips between receptive field centers and the sensitivity surface becomes bumpy. C, The Fourier transform of the sensitivity surface of the array shows that flatness breaks abruptly for receptive field spacing exceeding $2 \sigma . y$-Axis shows modulation amplitude at the fundamental frequency divided by the mean.

on one cell, adding the response of the other did not improve detection (Fig. $5 A, B$ ).

For a spot between the two cells, detection thresholds based on individual cell responses were higher than for centered spots (5.5 and 9.9\%) (Fig. 5C). However, threshold computed from the combined responses matched the average of the thresholds for a spot located directly over the individual cells (mean difference of $<5 \%)$ (Fig. $5 E)$, and this was true for all recorded pairs $(n=15)$ (Fig. 5D-F). Thus, also at threshold, the contrast sensitivity surface was flat (Fig. $5 F$ ).

These data show that detection of a threshold spot over one cell is not improved by including the response of a neighbor (Fig. $5 A, B)$. This was primarily because the response of the neighbor was weak, with a low SNR (Fig. 6A), but each cell has several neighbors, and conceivably their pooled responses might help. To test this, we used the recorded responses to simulate additional neighbors by assigning to each one the successive responses of the recorded neighbor. This simulation assumed that neighbors have identical response characteristics (i.e., belong to the same cell type) and that noise in their responses is uncorrelated. When up to six neighbors were added, contrast detection still did not improve (Fig. 6B). As a control, we assigned neighbors the successive responses of the optimally stimulated center cell; then, as expected, detection did improve, as the square root of the number of cells (Fig. 6B).

\section{Combined sensitivity of $\mathrm{ON}$ and OFF arrays}

Within each brisk-transient array ( $\mathrm{ON}$ and OFF), cells were separated by $\sim 200 \mu \mathrm{m}$. We selected several pairs across arrays (ON/OFF) with the same separation and stimulated with a spot of either polarity. Like the same-sign pairs, neighbors in an ON/OFF pair did not improve contrast detection for a spot centered on either cell (Fig. 5D). Furthermore, for a spot between cells, combined threshold matched the average for the two cells with centered spots (Fig. 5E). For one ON/OFF pair with relatively high receptive field overlap $(\sim 74 \%$ shared excitatory receptive field area, center spacing $1.2 \sigma$ ), combined threshold was somewhat lower than that for either cell alone (Fig. 5E, marked with ${ }^{\star}$ ).

For the foregoing experiments, we deliberately selected pairs separated by $\sim 200$ $\mu \mathrm{m}$. However, because $\mathrm{ON}$ and $\mathrm{OFF}$ arrays cover the retina independently, $\mathrm{ON}$ and OFF receptive fields can overlap completely. Furthermore, an ON cell could respond to a dark flash with a decrease in spontaneous firing, which could improve detection. To test the combined responses of co-spatial ON and OFF receptive fields, we combined in simulation the response of an ON cell to a centered dark spot with the response of an OFF cell to the same spot. Surprisingly, this yielded the same detection threshold as the OFF cell response alone.

To explain this, we assessed the spontaneous firing rates of the cells. These were generally low: $0.3 \pm 0.2$ spikes/s for OFF cells $(n=24$; mean \pm SEM) and $7.5 \pm 2.5$ spikes $/ \mathrm{s}$ for ON cells $(n=17)$. Although ON cells on average showed higher spontaneous firing than OFF cells, 11 of 17 ON cells had rates $<4.0$ spikes/s. With trial-to-trial response variability, it appears that such a low spike count cannot give reliable detection through a decrease in firing. Although we cannot exclude that an ON cell with high spontaneous rate could improve OFF cell detection of a dark flash, we found no such effect in our data. Thus, although the off response of an ON array might signal a suprathreshold dark spot, this response does not improve detection at threshold.

\section{Optimal spacing in a ganglion cell array}

The $\sim 2 \sigma$ spacing of receptive field centers is conserved across mammalian ganglion cell arrays. This suggests that this cell separation is functionally meaningful, and indeed, we found that, for small spots, this spacing provides uniform contrast sensitivity with the fewest cells. However, under natural viewing conditions, the input of the retina is more complex and has a distinct statistical structure: $1 / f$ spatial correlations (Field, 1987) and a skewed intensity distribution (Richards, 1982). Because these properties were instrumental in explaining the structure of the spatiotemporal receptive field of an individual cell (Laughlin, 1983; Atick and Redlich, 1990; Tsukamoto et al., 1990), we asked, how well is an array with $2 \sigma$ spacing adapted for representing information from natural scenes? 

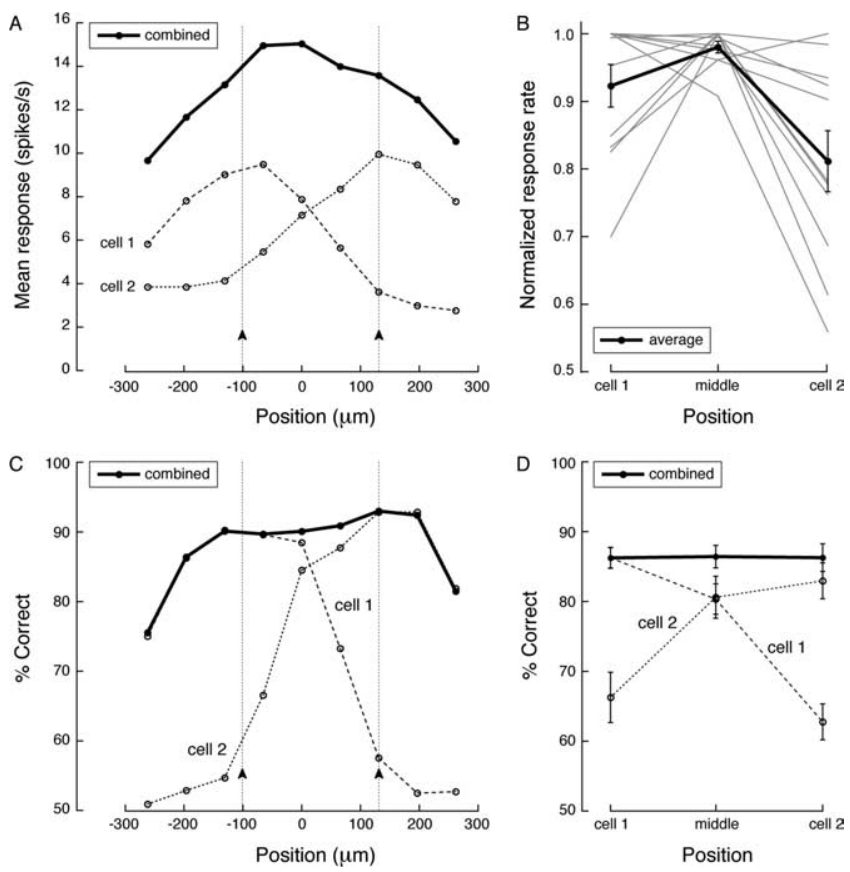

Figure 4. Simultaneous recordings from neighboring ganglion cells confirm that contrast sensitivity is spatially invariant. A spot was presented at nine locations along a line through the receptive field centers of a brisk-transient $0 \mathrm{~N} / 0 \mathrm{~N}$ ganglion cell pair. $\boldsymbol{A}$, At suprathreshold contrast (3.2\%), the firing rate of a cell increased when the spot was presented closer to its receptive field center (average rate during the $500 \mathrm{~ms}$ trial). The sum of the firing rates of the cells varied with spot position and peaked for a spot midway between the two cells. $\boldsymbol{B}$, This was true for all recorded pairs. $\boldsymbol{C}$, An ideal observer detected the spot based on the response of either cell, or both. When the spot was located near a receptive field center (arrows), detection performance for single and combined responses was the same. For locations between cells, detection based on the response of a single cell was worse than for a spot presented over the receptive field center of the cell. However, when the responses of the two cells were combined, ideal observer detection of a spot between receptive fields was the same as for a spot presented to the receptive field center of either cell. $\boldsymbol{D}$, This was observed for all pairs: combining responses of neighboring cells, percentage correct detection is the same at each spot position.

Using a model array (see Materials and Methods), we tested with natural images how spacing affects information represented in array responses (Fig. 7A) (van Hateren and van der Schaaf, 1998). We found for a fixed density of array elements that, as receptive field centers expanded, their SNRs improved because of summation over multiple correlated signals. Improving SNR increased the information represented in each receptive field. Conversely, larger receptive field centers also gave greater receptive field overlap, which increased redundancy between array elements. This, in turn, reduced total information represented by the array. To evaluate this tradeoff, we assessed the optimal overlap.

The fixed separation of the model ganglion cell receptive fields was expressed in terms of $\sigma$, the SD of the center Gaussian. Larger spacing in terms of $\sigma$ indicates less receptive field overlap. The model showed that represented information peaked when the spacing was $1.9 \sigma(\mathrm{ON})$ and $1.8 \sigma(\mathrm{OFF})($ Fig. $7 B)$. Thus, optimal overlap was slightly greater for the OFF array than for the $\mathrm{ON}$ array, and the range of measured receptive field spacings (ON, $2.05 \pm 0.50 \sigma$; OFF, $1.86 \pm 0.55 \sigma)$ tightly bracketed the computed optimum. Importantly, the optimum lay far from the $\sim 4 \sigma$ spacing required for simple tiling without overlap of the excitatory region of the receptive fields.

Although the amount of represented information (height of the curves in Fig. $7 B$ ) is expected to vary with the assumed re- sponse distribution, the location of the optimum is not. This is because the optimum depends primarily on how the amount of information changes with receptive field size. Because SNR improves by summation over additional cones, in any model, the amount of represented information will improve as the logarithm of the receptive field diameter (Derrington and Lennie, 1984) (see Materials and Methods, Eq. 5). Our model provides a simple embodiment of this general phenomenon.

The hypothesized advantage of overlap is that it allows individual cells to expand their centers and so improve their SNR by averaging spatially correlated cone signals. This implies that overlap would confer no significant advantage for images lacking spatial correlations. To test this, we studied the optimal array for representing spatial white noise, which by definition lacks correlations (Fig. 7A, right inset). The separation of receptive field centers was fixed, whereas receptive field widths (hence overlap) varied. As overlap decreased (and spacing expressed as $\sigma$ increased), the total represented information gradually increased (Fig. $7 B$ ). Thus, the optimal spacing minimized receptive field overlap, but, because the rate of increase was slow, there was little advantage to selecting a particular array spacing provided it was greater than $\sim 2 \sigma$. This suggests that the strongly conserved $2 \sigma$ spacing of ganglion cell arrays is a specific adaptation to the statistical regularities of natural images.

To test what properties of natural scenes set the optimal spacing, we studied the optimal array for representing natural pink noise: synthetic images with both the $1 / f$ power spectrum (Field, 1987) and the skewed intensity distribution (Richards, 1982) of natural scenes. For these synthetic images, the amount of information represented by the array was greater than for natural scenes, because natural pink noise, lacking higher-order correlations, is less redundant. Nevertheless, the optimal array spacing for the synthetic images was identical to that for natural scenes $(1.9 \sigma$ for model ON receptive fields) (Fig. $7 B)$. This demonstrated that the skewed intensity distribution and $1 / f$ power spectrum of natural images were sufficient to explain the optimal array spacing.

Measured with a white-noise stimulus, the inhibitory surround of a brisk-transient cell was typically small and weak (width, $1.37 \sigma$ for $\mathrm{ON}$ and $1.40 \sigma$ for OFF; strength relative to the surround, 0.81 for ON and 0.72 for OFF). This agrees with previous reports for white-noise stimuli (Chichilnisky, 2001; Chichilnisky and Kalmar, 2002), whereas stimuli containing spatial correlations (e.g., sine waves) evoke a larger and stronger surround (Derrington and Lennie, 1982). Because natural scenes have spatial correlations, the latter should be true also for natural stimuli. We therefore tested how relative surround width affects optimal arrangement of receptive fields.

As surround width increased relative to center width, optimal overlap decreased (i.e., optimal spacing expressed as $\sigma$ increased) (Fig. 7C). This can be explained by observing that larger surrounds are less correlated with their centers and therefore less effectively reduce the redundancy of neighboring ganglion cell signals. In the optimal array, this is compensated by decreasing receptive field overlap, or equivalently, increasing the spacing in terms of $\sigma$. Nevertheless, the effect on the optimal array geometry was small: with a twofold wider surround, optimal spacing remained within the range of spacings measured in the retina. Moreover, across the range of tested surround widths $(1.25-5 \sigma)$, the optimal array always showed substantial receptive field overlap and never showed perfect tiling of the excitatory centers. 
A
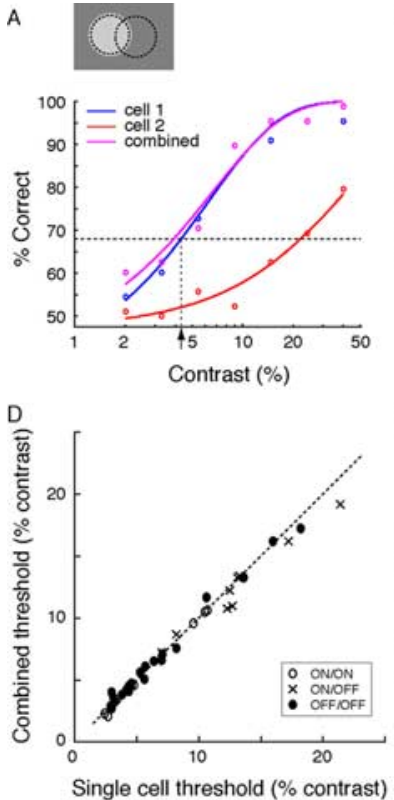

B
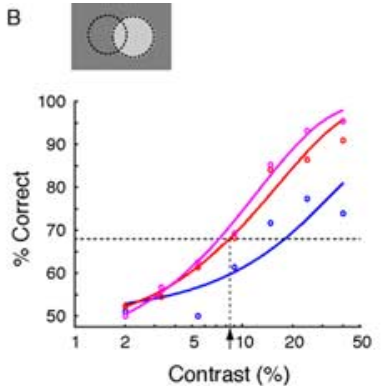

E

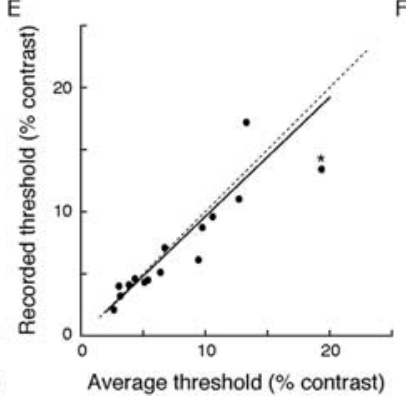

C
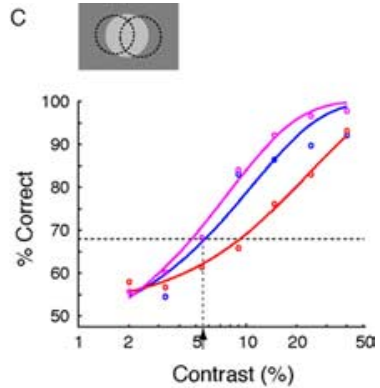

F

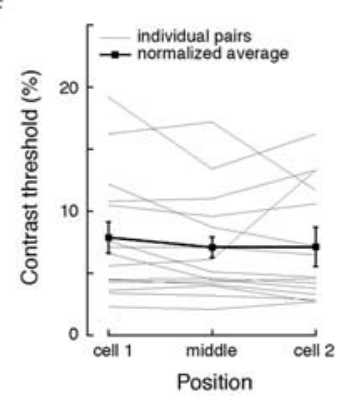

Figure 5. Single-cell threshold for contrast detection does not improve by adding the response of a neighbor. Recording from a ganglion cell pair, a spot of optimal size was presented over the center or midway between either cell. An ideal observer detected the spot based on the response of either cell alone, or combined. The task of the ideal observer was to detect nonzero contrast in a single-interval, two-alternative, forced-choice paradigm. $\boldsymbol{A}, \boldsymbol{B}$, Graphs show detection performance as the percentage correct choices on the basis of the response of cell 1, cell 2, or both. The dotted line represents detection threshold, set at $68 \%$ correct. For a spot centered on one cell, contrast detection was not improved by including the response of the other cell. $\boldsymbol{C}$, For a spot midway between the two cells, both contribute to detection, but threshold is similar to that for a spot centered on either cell. $\boldsymbol{D}-\boldsymbol{F}$, Combined contrast thresholds are as low as the best single-cell threshold and constant over space. $\boldsymbol{D}$, Contrast threshold based on the best single-cell response was the same as that based on the combined responses of neighbors. $\boldsymbol{E}$, Moreover, the average of the thresholds of the two cells for a centered spot and the recorded threshold for a spot located between them differed by $<5 \%$ (solid line; linear fit, slope 0.96). Data point marked with * represents an ON/OFF pair with unusually high overlap (for details, see Results). $\boldsymbol{F}$, Combined contrast threshold was the same for spots centered on and between cells. Thick line shows mean $\pm 1 S D$
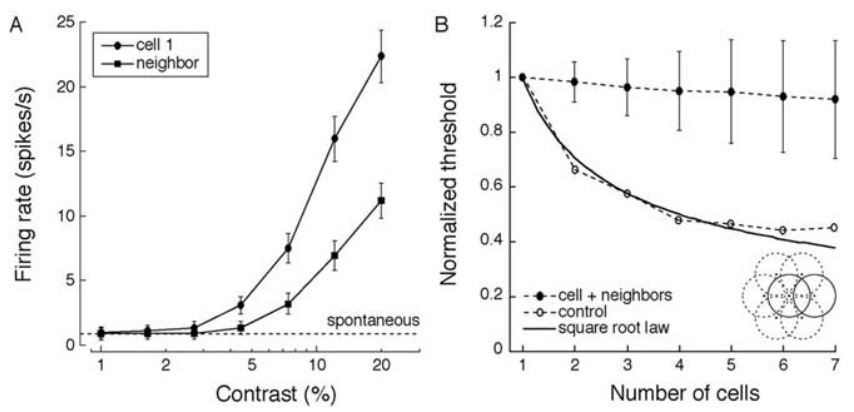

Figure 6. Simulated contributions of all neighbors do not improve contrast detection. $\boldsymbol{A}$, For a centered spot at threshold ( $\sim 4 \%$ contrast; $0 \mathrm{FF} / 0 \mathrm{FF}$ pair), a neighbor fires $<2$ spikes $/ \mathrm{s}$, which suggests minimal redundancy. $\boldsymbol{B}$, We recorded responses from pairs of neighboring ganglion cells (filled circles) with a spot centered on one cell. Contrast threshold, computed with an ideal observer, does not improve when the responses of the center cell (inset, solid line) and a single neighbor (inset, dotted line) are combined (mean \pm 1 SD). We simulated additional neighbors (inset, dotted lines) from the response of the recorded neighbor (see Results). For all recorded pairs ( $n=15$ ), adding up to six neighbors did not significantly lower the threshold for contrast detection ( $t$ test, $p=0.13$ ). In a control simulation, combining successive responses from the center cell improved detection in accordance with the square root law (open circles).

\section{Robustness of the model prediction}

Our model used an estimated cone SNR of $\sim 100$ (see Materials and Methods). To test the sensitivity of our results to the accuracy of this estimate, we computed the optimal spacing of ON and OFF arrays while varying the cone SNR from 1 to $10^{4}$. Across this range, the amount of information signaled by the array increased as the logarithm of the SNR (data not shown). However, optimal spacing varied little, from 1.5 to $2 \sigma$ (Fig. $7 D$ ), which was well within the range of spacings measured in the guinea pig retina (Fig. $2 B$ ). Although our model used existing data to derive a best estimate of the cone SNR, this result shows that predicted optimal cell spacing is robust to substantial deviations in the real cone SNR. In summary, optimal overlap depends on balancing SNR improvement attributable to receptive field expansion against redundancy attributable to receptive field overlap. Optimal overlap is primarily insensitive to absolute SNR in the cone and to the ganglion cell response function and center-surround receptive field dimensions.

\section{Discussion \\ Functional architecture of ganglion cell arrays}

Dendritic fields of neighboring ganglion cells were initially thought to tile the retina efficiently, i.e., to completely cover it with minimal overlap (Wassle et al., 1975, 1981a; Peichl and Wassle, 1979). This "territoriality" was thought to be achieved through mutual dendritic repulsion (Wassle et al., 1981a; Perry and Linden, 1982). However, in $\mathrm{Brn}_{3} \mathrm{~b}^{-/-}$mice, in which $\sim 80 \%$ of ganglion cells die early in development, there was no increase in dendritic field size for the surviving cells but instead gaps in dendritic coverage (Lin et al., 2004). Moreover, although some types do tile (Dacey, 1989; Vaney, 1994), the dendritic tips of a cell typically reach the neighboring somas as shown here for guinea pig (Fig. 2A) and elsewhere for cat (Wassle et al., 1981b; Cohen and Sterling, 1992; Stein et al., 1996), ferret (Lohmann and Wong, 2001), and primate (Dacey and Brace, 1992). Thus, tiling is a special case and dendritic overlap (coverage factor of $\sim 3-4$ ) is the rule (Wassle, 2004).

We can now reconcile this degree of dendritic overlap with other aspects of the functional architecture of an array. As shown first for model arrays of linearly summed Gaussian receptive fields (DeVries and Baylor, 1997) and here for actual neurons with their nonlinearities and response variability, $2 \sigma$ spacing of the domed receptive field centers is just sufficient to provide a flat contrast sensitivity surface. This is true for near-threshold as well as strongly suprathreshold stimuli (Figs. 4-6). However, it remained to explain precisely why sensitivity should be flat and what fundamental property sets the optimal degree of overlap. To explore this, we modeled how represented information is affected by array structure.

Knowing that dendritic structure optimally improves SNR for partially correlated signals to maximize information in a single cell (Tsukamoto et al., 1990), we reasoned that array structure might maximize total information in an array. This hypothesis followed a pioneering effort to calculate for model photoreceptor arrays tradeoffs between acuity, sensitivity, and total information (Snyder et al., 1977). However, that study used white noise as input, which seemed inappropriate here because white noise 
lacks the natural spatial correlations to which individual cells are adapted. Indeed, classic work showed that center-surround receptive field architecture optimizes information transmission from natural images rather than white noise (Srinivasan et al., 1982; Atick and Redlich, 1990; van Hateren, 1992). Therefore, we tested model arrays with natural images and used white noise as a control.

It transpired that an array is optimal for natural images when cells extend their dendrites to improve SNR but balance this against the redundancy caused by the added overlap. Greater SNR increases information, but greater redundancy (by occupying more of the limited dynamic range of each cell) reduces information. For natural images, these tendencies are balanced at an array spacing of $\sim 2 \sigma$. For white noise, the total represented information increases steadily with decreasing overlap because, lacking correlations, the representation of white noise is not improved by summation. Thus, for white noise, an array is strictly optimal when receptive field overlap is minimal. This null result for white noise strengthens the broad hypothesis that the spatial structure of a ganglion cell array is adapted to the statistics of natural scenes.

We calculated the hypothesized tradeoff between SNR improvement and redundancy in terms of a common currency: bits of information. However, redundantly encoded information also has a concrete cost in retinal resources: representing more information requires more synapses within the retina (C. Ratliff, H. Kao, P. Sterling, and V. Balasubramanian, unpublished observations) (Sterling, 2004) and more spikes at the output (Koch et al., 2006), which makes redundant informa-

tion expensive (Levy and Baxter, 1996; Attwell and Laughlin, 2001; Balasubramanian et al., 2001; de Polavieja, 2004; Koch et al., 2006). Interestingly, minimizing the energetic cost of signaling in a simple model can produce arrays resembling retinal mosaics (Vincent and Baddeley, 2003).

\section{Flat sensitivity surface versus maximizing information}

Maximizing total information represented by an array does not require that the array elements communicate equal information about each point in a visual scene. Indeed, we find that reducing redundancy at the expense of SNR improvement is not identical to achieving a flat sensitivity surface. Specifically, for model receptive field surrounds of more than twice the measured width, optimal spacing is $>3 \sigma$ (Fig. $7 C$ ). For such spacings, the linearly summed contrast sensitivity surface is not flat (Fig. $3 A, C$ ). Thus, for the two alternative hypotheses concerning receptive field overlap (i.e., flat sensitivity vs maximum information) to agree, the relative surround width must lie within a specific range, and that is where our measurements fell (Fig. 7C).
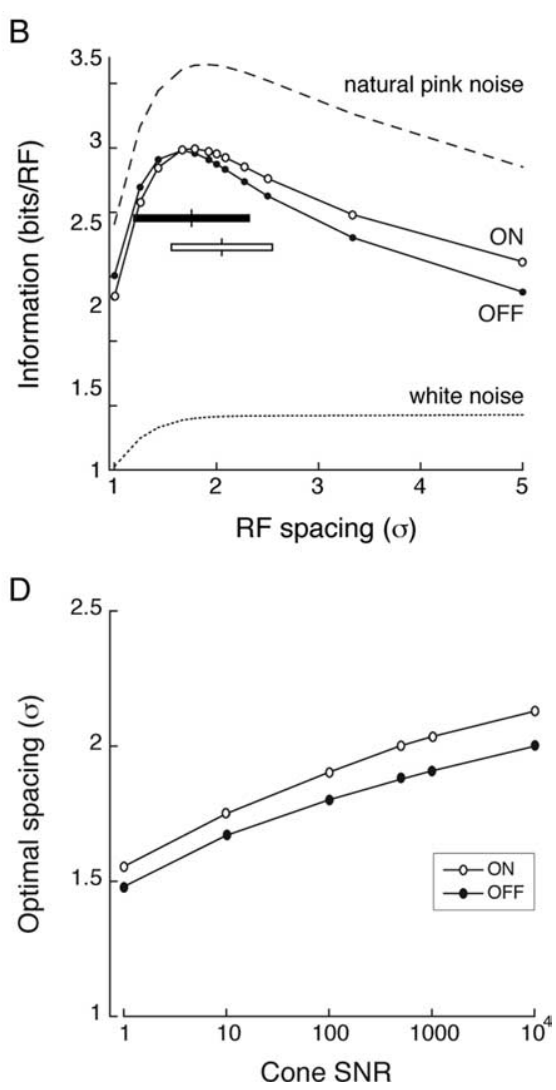

Figure 7. Information about natural scenes is maximized for model arrays when receptive fields are spaced at approximately natural images. Left inset, Small patch of natural image with overlaid array. Right inset, Small patch of white noise with an orla array. $\boldsymbol{B}$, Information represented from natural images peaks at a receptive field (RF) spacing of $\sim 2 \sigma$. Bars show the spacing \pm 1 SD for the $0 \mathrm{~N}$ and $0 \mathrm{FF}$ arrays ( $0 \mathrm{~N}, n=8$, light bar; $0 \mathrm{FF}, n=12$, dark bar). Tested with synthetic inages (natural pink noise; see Results), represented information peaks at the same receptive field spacing as for natural

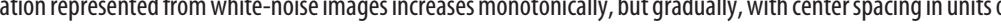
is robust to changes in receptive field surround width: a twofold increase in the surround width would leave optimal spacing would lead to widely spaced optimal arrays ( $>3 \mathrm{~s}$ ) with contrast sensitivity surfaces that are not flat (compare Fig. $3 \mathrm{~A}$, C; see Discussion). D, Optimal spacing is also robust to changes in the cone SNR estimate: over four orders of magnitude of the SNR, optimal array spacing remains within the measured range.

\section{Mechanisms for independent signaling}

The redundancy accompanying $2 \sigma$ receptive field spacing implies that neighboring cells do not encode information entirely independently. To quantify the degree of (in)dependence, we calculated the amount of information conveyed by a cell and by its neighbor concerning the presence of a stimulus. Perfect detection corresponds to 1 bit of information. Correct detections with probability $p$ correspond to $I=1+p \log _{2} p+(1-p) \log _{2}(1-$ $p)$ bits. For a cell with centered suprathreshold stimulus, $p$ is $\sim 0.98$, whereas for the neighbor, $p$ is $\sim 0.85$. For the cell with centered threshold stimulus, $p=0.68$ (by definition), whereas for the neighbor, $p$ is $\sim 0.54$. Thus, above threshold, the ratio of information carried by the centered cell and its neighbor was $\sim 2$, whereas at threshold, the ratio was $\sim 20$. Thus, a ganglion cell and its neighbor signal more independently at threshold than at high contrast, in agreement with previous reports (Mastronarde, 1983; Meister et al., 1995; Schnitzer and Meister, 2003). Thus, the encoding of contrast of an array is sparse near threshold and more redundant at high contrast.

Given a dendritic coverage factor of 3-4 and receptive field 
center coverage factor of $4-6$ (Fig. $2 A, B$ ), how can a ganglion cell respond independently of its neighbors? Several mechanisms probably contribute. First, the Gaussian distribution of synapses ensures that a spot centered on one ganglion cell excites approximately threefold more bipolar cell synapses on that cell than on any one of its neighbors (Fig. 2 B). Consequently, a stimulus just strong enough to evoke spikes in the optimally stimulated cell is too weak (by approximately threefold) to reliably evoke spikes from a neighbor. Second, a stimulus centered on one cell falls substantially on the surround of the neighbor, causing lateral inhibition. Third, a presynaptic, nonlinear mechanism combines with the spike threshold to prevent transmission of the smaller graded signals (Zaghloul et al., 2003; Dhingra and Smith, 2004). Thus, to a center spot near threshold, a ganglion cell fires relatively independently because local circuits and cellular mechanisms selectively attenuate weaker signal of the neighboring cells.

\section{Predictions for other neuronal arrays}

Our finding that array structure maximizes information from spatially correlated signals makes several predictions. (1) Arrays should adjust their overlap to the particular aspect of a natural scene for which they are specialized, e.g., bright versus dark regions. This might explain the modest difference in overlap between $\mathrm{ON}$ and OFF arrays (Figs. 2B, 7B) (Ratliff, Kao, Sterling, and Balasubramanian, unpublished observations). (2) Overlap should be strongly conserved across mammals that share the same terrestrial environment. It is (Peichl, 1991). (3) Overlap should differ for animals in habitats with different power spectra, for example, terrestrial versus aquatic (Atick et al., 1992; Balboa and Grzywacz, 2003), which can be tested.

The insight regarding array structure (maximize information by balancing SNR improvement against redundancy) can be applied to predict overlap for any neuronal array, given the receptive field weighting profile and array density of its cells. Although most receptive fields are dome-like, with $\sim 2 \sigma$ spacing (such as those studied here) (DeVries and Baylor, 1997), two exceptions are known. ON-OFF directionally selective ganglion cells tuned to the same preferred direction show simple tiling without overlap (Vaney, 1994), and so do midget ganglion cells (Dacey, 1994). The explanation for directionally selective cells, which are selective for motion, might involve considering temporal correlations: neighbors with the same directional tuning should not overlap because a preferred stimulus moving from one cell to next evokes temporally correlated responses that would be more informative if the receptive fields did not overlap spatially. The explanation for midget cells might reside in the specific spatial weighting function of these cells: for a fixed distance between cells, predicted optimal spacing increases for cells with a relatively broad surround (Fig. 7C).

More generally, our methods can be used to assess how effectively the tuning functions of individual cells within any array are distributed across the dynamic range of the input of the array. This addresses a fundamental question about the neuronal representation of the sensory environment, a question that is relevant beyond the arrays of the retina and even beyond the visual system per se. The model here described could be used to study the neuronal arrays that encode color, orientation, and direction of motion in the visual cortex, and even place cells in the hippocampus.

\section{Appendix}

\section{Information in receptive field arrays}

To compute the redundancy in receptive field arrays, we first recognized that the response of our model receptive fields can be split into a weighted sum of local luminance $\left(L_{c}\right.$, the central Gaussian response) and contrast (balanced center - surround):

Receptive field $=L_{c}-k L_{s}=(1-k) L_{c}+k\left(L_{c}-L_{s}\right)=L+C$.

Here $k$ is the surround weighting and $C=k\left(L_{c}-L_{s}\right)$ is the contrast contribution to the receptive field. Luminance and contrast are statistically independent in natural scenes (Mante et al., 2005). Because of this statistical independence, the information in the responses of the receptive field arrays $\left(I_{N}\right)$ is bounded from below by the information in the contrast array (Madiman and Barron, 2008) and is bounded from above by the sum of the information in luminance and the information in contrast (Cover and Thomas, 1991):

$$
I(C) \leq I_{N} \leq I(C)+I(L) .
$$

The luminance in natural scenes is well known to have scaleinvariant correlations that persist over the entire scene (Burton and Moorhead, 1987; Field, 1987), whereas contrast correlations decay rapidly with distance (Srinivasan et al., 1982; Atick and Redlich, 1990).

The information in an $m \times m$ array of luminance filters (central Gaussians) can be written as

$$
I_{m \times m}=m^{2} I_{1}(1-\delta),
$$

where $I_{1}$ is the luminance information in a single filter and $\delta$ measures the redundancy in the $m \times m$ array. The scale invariance of the correlations in luminance further implies that

$$
I_{m}^{k} \times m^{k}=m^{2 k} I_{l}(1-\delta)^{k} .
$$

Letting $N$ be the total number of receptive fields in the array, we can write this as

$$
I_{N}=N \cdot I_{1}(1-\delta)^{\frac{1}{2} \log _{m} N}=N^{(1-\varepsilon)} \cdot I_{1}
$$

with

$$
0<\varepsilon=-\frac{1}{2} \log _{m}(1-\delta)<1 .
$$

Thus, the information in luminance scales sublinearly with the number of elements in the array.

However, the information in contrast scales linearly with the number of elements in the array because the correlations decay rapidly with distance (Fig. 6C). Thus, for large arrays, we can write

$$
I(C) \leq I_{N} \leq I(C)\left(1+O\left(N^{-\varepsilon}\right)\right)
$$

implying that, when $N$ is large, the information in contrast gives a very good estimate of the information in the full receptive field array response. In what follows, we will therefore take $I(C) \approx I_{N}$.

Contrast correlations are primarily the result of overlap between nearest neighbor receptive fields (Meister, 1996; DeVries, 1999; Meister and Berry, 1999). Thus, the shared information between contrast filters (balanced center - surround) is negligible for all but nearest neighbors. Therefore, we can write an approximate formula for the information in a rectangular contrast 


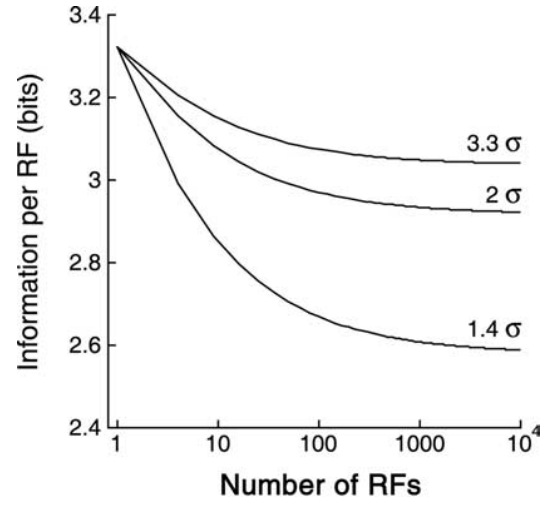

Figure 8. Information per receptive field asymptotes to a constant for large arrays. Given a fixed number of response levels (SNR), the asymptotic value is lower for arrays with greater overlap (smaller spacing in terms of $\sigma$; spacings of $3.3 \sigma, 2 \sigma$, and $1.4 \sigma$ shown). This reflects redundancy in responses of overlapping receptive fields. Here, contrasts were quantized to 10 levels, matching the dynamic range and variability of ganglion cell responses reported in the literature (see Appendix).

array in terms the of the number of adjacent neighbors $\left(N_{\text {adj }}\right)$ and the number of diagonal neighbors $\left(N_{\text {diag }}\right)$ :

$$
I_{N}=N I_{l}-N_{\text {adj }} M I_{\text {adj }}-N_{\text {diag }} M I_{\text {diag }}
$$

where $M I_{\text {adj }}$ is the mutual information (for a definition of mutual information, see Cover and Thomas, 1991) between adjacent pairs (and similarly for diagonals). To test this formula, we constructed $2 \times 3$ arrays of contrast filters quantized to 10 equally likely response levels, and $3 \times 3$ arrays quantized to 4 response levels; responses were rectified so that model ON cells represented positive contrast, whereas model OFF cells represented negative contrast. Quantization of the response models the distinguishable signaling levels of a ganglion cell after accounting for noise (Dhingra and Smith, 2004). Mapping responses to 10 levels in this way requires a saturating nonlinearity, thus including a simple model of the nonlinearity in the ganglion cell response. In each case, the formula predicted the measured joint entropy to within $1.5 \%$ at a separation of $2 \sigma$. Figure 8 shows how contrast information per receptive field (i.e., $I_{N} / N$ with $I_{N}$ given by the formula above) changes with $N$ for different array spacings. In all cases, the information per receptive field for very large arrays asymptotes to a constant value that depends on the cell spacing and geometry of the array. For large rectangular arrays containing $N$ receptive fields, the number of adjacent and diagonal pairs $\left(N_{\text {adj }}, N_{\text {diag }}\right)$ can be counted explicitly. The total information in a receptive field array is then given by

$$
I_{N} \approx N\left(I_{1}-2 M I_{\text {adj }}-2 M I_{\text {diag }}\right),
$$

matching the asymptotic values for large $N$ in Figure 8.

\section{References}

Adelson EH (1982) Saturation and adaptation in the rod system. Vision Res 22:1299-1312.

Atick JJ, Redlich AN (1990) Towards a theory of early visual processing. Neural Comput 2:308-320.

Atick JJ, Li Z, Redlich AN (1992) Understanding retinal color coding from first principles. Neural Comput 4:559-572.

Attwell D, Laughlin SB (2001) An energy budget for signaling in the grey matter of the brain. J Cereb Blood Flow Metab 21:1133-1145.

Balasubramanian V, Kimber D, Berry II MJ (2001) Metabolically efficient information processing. Neural Comput 13:799-815.

Balboa RM, Grzywacz NM (2003) Power spectra and distribution of contrasts of natural images from different habitats. Vision Res 43:2527-2537.

Barlow HB (1961) Possible principles underlying the transformations of sensory messages. In: Sensory communication (Rosenblith WA, ed), pp 782-786. Cambridge, MA: MIT.

Braverman D (1962) Learning filters for optimum pattern recognition. IRE Transact Inform Theory 8:280-285.

Burton GJ, Moorhead IR (1987) Color and spatial structure in natural scenes. Appl Opt 26:157-170.

Calkins DJ, Sterling P (2007) Microcircuitry for two types of achromatic ganglion cell in primate fovea. J Neurosci 27:2646-2653.

Chichilnisky EJ (2001) A simple white noise analysis of neuronal light responses. Netw Comput Neural Syst 12:199-213.

Chichilnisky EJ, Kalmar RS (2002) Functional asymmetries in ON and OFF ganglion cells of primate retina. J Neurosci 22:2737-2747.

Chichilnisky EJ, Kalmar RS (2003) Temporal resolution of ensemble visual motion signals in primate retina. J Neurosci 23:6681-6689.

Chichilnisky EJ, Rieke F (2005) Detection sensitivity and temporal resolution of visual signals near absolute threshold in the salamander retina. J Neurosci 25:318-330.

Choi SY, Borghuis BG, Rea R, Levitan ES, Sterling P, Kramer RH (2005) Encoding light intensity by the cone photoreceptor synapse. Neuron 48:555-562.

Cohen E, Sterling P (1992) Parallel circuits from cones to the On-beta ganglion cell. Eur J Neurosci 4:506-520.

Cover TM, Thomas JA (1991) Elements of information theory. New York: Wiley.

Dacey DM (1989) Monoamine-accumulating ganglion cell type of the cat's retina. J Comp Neurol 288:59-80.

Dacey DM (1994) Physiology, morphology and spatial densities of identified ganglion cell types in primate retina. Ciba Found Symp 184:12-28; discussion 28-34:63-70.

Dacey DM, Brace S (1992) A coupled network for parasol but not midget ganglion cells in the primate retina. Vis Neurosci 9:279-290.

de Polavieja GG (2004) Reliable biological communication with realistic constraints. Phys Rev E Stat Nonlin Soft Matter Phys 70:061910.

DeAngelis GC, Ohzawa I, Freeman RD (1995) Receptive-field dynamics in the central visual pathways. Trends Neurosci 18:451-458.

Demb JB, Haarsma L, Freed MA, Sterling P (1999) Functional circuitry of the retinal ganglion cell's nonlinear receptive field. J Neurosci 19:9756-9767.

Derrington AM, Lennie P (1982) The influence of temporal frequency and adaptation level on receptive field organization of retinal ganglion cells in cat. J Physiol (Lond) 333:343-366.

Derrington AM, Lennie P (1984) Spatial and temporal contrast sensitivities of neurones in lateral geniculate nucleus of macaque. J Physiol (Lond) 357:219-240.

DeVries SH (1999) Correlated firing in rabbit retinal ganglion cells. J Neurophysiol 81:908-920.

DeVries SH, Baylor DA (1997) Mosaic arrangement of ganglion cell receptive fields in rabbit retina. J Neurophysiol 78:2048-2060.

DeVries SH, Li W, Saszik S (2006) Parallel processing in two transmitter microenvironments at the cone photoreceptor synapse. Neuron 50:735-748.

Dhingra NK, Smith RG (2004) Spike generator limits efficiency of information transfer in a retinal ganglion cell. J Neurosci 24:2914-2922.

Dhingra NK, Kao YH, Sterling P, Smith RG (2003) Contrast threshold of a brisk-transient ganglion cell in vitro. J Neurophysiol 89:2360-2369.

Duda RO, Hart PE, Stork DG (2000) Pattern classification. New York: Wiley Interscience.

Emoto K, Parrish JZ, Jan LY, Jan YN (2006) The tumour suppressor Hippo acts with the NDR kinases in dendritic tiling and maintenance. Nature 443:210-213.

Field DJ (1987) Relations between the statistics of natural images and the response properties of cortical cells. J Opt Soc Am A 4:2379-2394.

Field GD, Chichilnisky EJ (2007) Information processing in the primate retina: circuitry and coding. Annu Rev Neurosci 30:1-30.

Frechette ES, Sher A, Grivich MI, Petrusca D, Litke AM, Chichilnisky EJ (2005) Fidelity of the ensemble code for visual motion in primate retina. J Neurophysiol 94:119-135.

Freed MA, Smith RG, Sterling P (1992) Computational model of the onalpha ganglion cell receptive field based on bipolar cell circuitry. Proc Natl Acad Sci USA 89:236-240.

Geisler WS (1989) Sequential ideal-observer analysis of visual discriminations. Psychol Rev 96:267-314. 
Geisler WS, Albrecht DG, Salvi RJ, Saunders SS (1991) Discrimination performance of single neurons: rate and temporal-pattern information. J Neurophysiol 66:334-362.

Kier CK, Buchsbaum G, Sterling P (1995) How retinal microcircuits scale for ganglion cells of different size. J Neurosci 15:7673-7683.

Koch K, McLean J, Berry M, Sterling P, Balasubramanian V, Freed MA (2004) Efficiency of information transmission by retinal ganglion cells. Curr Biol 14:1523-1530.

Koch K, McLean J, Segev R, Freed MA, Berry II MJ, Balasubramanian V, Sterling P (2006) How much the eye tells the brain. Curr Biol 16:1428-1434.

Laughlin S (1994) Matching coding, circuits, cells, and molecules to signals - general-principles of retinal design in the fly's eye. Prog Retin Eye Res 13:165-196.

Laughlin SB (1983) Matching coding to scenes to enhance efficiency. In: Physical and biological processing of images (Braddick OJ, Sleigh AC, eds), pp 42-52. Berlin: Springer.

Levy WB, Baxter RA (1996) Energy efficient neural codes. Neural Comput 8:531-543.

Lin B, Wang SW, Masland RH (2004) Retinal ganglion cell type, size, and spacing can be specified independent of homotypic dendritic contacts. Neuron 43:475-485.

Lohmann C, Wong RO (2001) Cell-type specific dendritic contacts between retinal ganglion cells during development. J Neurobiol 48:150-162.

Madiman M, Barron AR (2007) Generalized entropy power inequalities and monotonicity properties of information. IEEE Trans Inf Theory, in press.

Mante V, Frazor RA, Bonin V, Geisler WS, Carandini M (2005) Independence of luminance and contrast in natural scenes and in the early visual system. Nat Neurosci 8:1690-1697.

Mastronarde DN (1983) Correlated firing of cat retinal ganglion cells. II. Responses of X- and Y-cells to single quantal events. J Neurophysiol 49:325-349.

Meister M (1996) Multineuronal codes in retinal signaling. Proc Natl Acad Sci USA 93:609-614.

Meister M, Berry II MJ (1999) The neural code of the retina. Neuron 22: 435-450.

Meister M, Lagnado L, Baylor DA (1995) Concerted signaling by retinal ganglion cells. Science 270:1207-1210.

Nakatani K, Tamura T, Yau KW (1991) Light adaptation in retinal rods of the rabbit and two other nonprimate mammals. J Gen Physiol 97:413-435.

Peichl L (1991) Alpha ganglion cells in mammalian retinae: common properties, species differences, and some comments on other ganglion cells. Vis Neurosci 7:155-169.

Peichl L, Wassle H (1979) Size, scatter and coverage of ganglion cell receptive field centres in the cat retina. J Physiol (Lond) 291:117-141.

Pelli DG (1997) The VideoToolbox software for visual psychophysics: transforming numbers into movies. Spat Vis 10:437-442.

Penn RD, Hagins WA (1972) Kinetics of the photocurrent of retinal rods. Biophys J 12:1073-1094.

Perry VH, Linden R (1982) Evidence for dendritic competition in the developing retina. Nature 297:683-685.

Richards W (1982) Lightness scale from image intensity distributions. Appl Opt 21:2569-2604.
Schnitzer MJ, Meister M (2003) Multineuronal firing patterns in the signal from eye to brain. Neuron 37:499-511.

Shlens J, Field GD, Gauthier JL, Grivich MI, Petrusca D, Sher A, Litke AM, Chichilnisky EJ (2006) The structure of multi-neuron firing patterns in primate retina. J Neurosci 26:8254-8266.

Snyder AW, Laughlin SB, Stavenga DG (1977) Information capacity of eyes. Vision Res 17:1163-1175.

Srinivasan MV, Laughlin SB, Dubs A (1982) Predictive coding: a fresh view of inhibition in the retina. Proc R Soc Lond B Biol Sci 216:427-459.

Stein JJ, Johnson SA, Berson DM (1996) Distribution and coverage of beta cells in the cat retina. J Comp Neurol 372:597-617.

Sterling P (2004) How retinal circuits optimize the transfer of visual information. In: The visual neurosciences (Chalupa LM, Werner JS, eds), pp 234-259. Cambridge, MA: MIT.

Tamura T, Nakatani K, Yau KW (1989) Light adaptation in cat retinal rods. Science 245:755-758.

Thomson EE, Kristan WB (2005) Quantifying stimulus discriminability: a comparison of information theory and ideal observer analysis. Neural Comput 17:741-778.

Tsukamoto Y, Smith RG, Sterling P (1990) “Collective coding” of correlated cone signals in the retinal ganglion cell. Proc Natl Acad Sci USA 87:1860-1864.

van Hateren JH (1992) Real and optimal neural images in early vision. Nature 360:68-70.

van Hateren JH (1993) Spatiotemporal contrast sensitivity of early vision. Vision Res 33:257-267.

van Hateren JH, van der Schaaf A (1998) Independent component filters of natural images compared with simple cells in primary visual cortex. Proc Biol Sci 265:359-366.

Vaney DI (1991) Many diverse types of retinal neurons show tracer coupling when injected with biocytin or Neurobiotin. Neurosci Lett 125:187-190.

Vaney DI (1994) Territorial organization of direction-selective ganglion cells in rabbit retina. J Neurosci 14:6301-6316.

Vincent BT, Baddeley RJ (2003) Synaptic energy efficiency in retinal processing. Vision Res 43:1283-1290.

Wassle H (2004) Parallel processing in the mammalian retina. Nat Rev Neurosci 5:747-757.

Wassle H, Boycott BB (1991) Functional architecture of the mammalian retina. Physiol Rev 71:447-480.

Wassle H, Levick WR, Cleland BG (1975) The distribution of the alpha type of ganglion cells in the cat's retina. J Comp Neurol 159:419-438.

Wassle H, Peichl L, Boycott BB (1981a) Dendritic territories of cat retinal ganglion cells. Nature 292:344-345.

Wassle H, Peichl L, Boycott BB (1981b) Morphology and topography of onand off-alpha cells in the cat retina. Proc R Soc Lond B Biol Sci 212:157-175.

Yin L, Smith RG, Sterling P, Brainard DH (2006) Chromatic properties of horizontal and ganglion cell responses follow a dual gradient in cone opsin expression. J Neurosci 26:12351-12361.

Zaghloul KA, Boahen K, Demb JB (2003) Different circuits for ON and OFF retinal ganglion cells cause different contrast sensitivities. J Neurosci 23:2645-2654. 